\title{
REPRESENTATIONS OF BANACH ALGEBRAS SUBORDINATE TO TOPOLOGICALLY INTROVERTED SPACES
}

\author{
M. FILALI, M. NEUFANG, AND M. SANGANI MONFARED
}

\begin{abstract}
Let $A$ be a Banach algebra, $X$ a closed subspace of $A^{*}, Y$ a dual Banach space with predual $Y_{*}$, and $\pi$ a continuous representation of $A$ on $Y$. We call $\pi$ subordinate to $X$ if each coordinate function $\pi_{y, \lambda} \in X$, for all $y \in Y, \lambda \in Y_{*}$. If $X$ is topologically left (right) introverted and $Y$ is reflexive, we show the existence of a natural bijection between continuous representations of $A$ on $Y$ subordinate to $X$, and normal representations of $X^{*}$ on $Y$. We show that if $A$ has a bounded approximate identity, then every weakly almost periodic functional on $A$ is a coordinate function of a continuous representation of $A$ subordinate to $W A P(A)$. We show that a function $f$ on a locally compact group $G$ is left uniformly continuous if and only if it is the coordinate function of the conjugate representation of $L^{1}(G)$, associated to some unitary representation of $G$. We generalize the latter result to an arbitrary Banach algebra with bounded right approximate identity. We prove the functionals in $L U C(A)$ are all coordinate functions of some norm continuous representation of $A$ on a dual Banach space.
\end{abstract}

\section{INTRODUCTION}

For a (not necessarily involutive) Banach algebra $A$, the close connections between representations of $A$ on the one hand, and elements of the dual space $A^{*}$ on the other hand, have been studied in several papers including Fell [17], Bonsall and Duncan [4,5], Young [38, Lau 28], Kaijser [26], Duncan and Ülger [15], and more recently, Runde 32 and Daws [12].

In this paper we shall continue the above studies by considering representations of $A$ whose coordinate functions belong to some topologically (left or right) introverted subspace $X$ of $A^{*}$ (for the undefined terminology used in the introduction, we refer to section 2). We shall call such representations subordinate to $X$.

The paper is organized as follows. In section 2 we review some basic definitions and preliminaries which are used throughout the paper. In section 3 we study the connections between representations of $A$ subordinate to $X$ and representations of $X^{*}$. The main result of this section (Theorem 3.3) shows that if $Y$ is a reflexive Banach space, then there exists a natural one-to-one correspondence between representations of $X^{*}$ on $Y$ and the representations of $A$ on $Y$ which are subordinate to $X$. This result can be regarded as an extension to non-involutive Banach algebras, of the well-known correspondence between the representations of a $\mathrm{C}^{*}$-algebra and the representations of its enveloping von Neumann algebra (see Remark 3.4(2)).

Received by the editors August 28, 2013.

2010 Mathematics Subject Classification. Primary 46H15, 46B10, 43A20, 47L10.

The second and third authors were partially supported by NSERC. 
It is known that for an involutive Banach algebra $A$ with bounded approximate identity, every positive linear functional on $A$ is a coordinate function of an involutive representation on some Hilbert space (see for example, Dixmier [14, Proposition 2.4.4]). Being a coordinate function of a representation on some Hilbert space, a positive linear functional is in fact weakly almost periodic by Young [38, page 102] or Filali-Monfared [18, Lemma 2.3].

Our aim in section 4 is to extend the result on positive linear functionals to weakly almost periodic functionals on $A$. We shall consider representations that are subordinate to the space of weakly almost periodic functionals $W A P(A)$. One of the earliest studies of a link between representations of $A$ and the weakly almost periodic functionals is the paper of Young [38]. Young proved that a normed algebra $A$ has a faithful continuous [isometric] representation on some reflexive Banach space if and only if the weakly almost periodic functionals of unit norm on $A$ separate the points of $A$ [comprise a norm-generating set] ([38, Theorem 1]). Also, implicit in the proof of the Young's theorem is the fact that all continuous representations of a Banach algebra on a reflexive Banach space are subordinate to $W A P(A)$ (cf. [38, page 102]). Our main result in section 4 is Theorem 4.4, which proves the converse of the latter result to the effect that if $A$ is a Banach algebra with a bounded approximate identity, then every weakly almost periodic functional on $A$ can be represented as a coordinate function of some continuous representation of $A$ on a reflexive Banach space.

Section 5 is devoted to a study of representations that are subordinate to $L U C(G)$ and $R U C(G)$, for $G$ a locally compact group, and more generally to $L U C(A)$ and $R U C(A)$, for $A$ a Banach algebra.

\section{Preliminaries}

We follow the standard terminology of Banach algebras and representation theory given, for example, in Palmer 31] or Dales [9. Suppose that $A$ is a Banach algebra and $Y$ is a Banach space and $\pi: A \longrightarrow \mathscr{L}(Y)$ is a representation. If $\pi$ is continuous with respect to the norm topologies of $A$ and $\mathscr{L}(Y)$, then we say that $\pi$ is a continuous representation. If both $A$ and $Y$ are dual spaces, and $\pi: A \longrightarrow \mathscr{L}(Y)$ is a continuous representation such that $\pi$ is also continuous in the $w^{*}$-topologies of $A$ and $\mathscr{L}(Y) \cong\left(Y \widehat{\otimes} Y_{*}\right)^{*}$, then we say that $\pi$ is a normal representation (here $Y_{*}$ denotes the predual of $Y$, and $\widehat{\otimes}$ denotes the projective tensor product; cf. Takesaki [36, Theorem IV.2.3 and Definition III.2.15]).

By a coordinate function of a representation $\pi: A \longrightarrow \mathscr{L}(Y)$ corresponding to $y \in Y$ and $\lambda \in Y^{*}$, we mean the linear functional $\pi_{y, \lambda}$ on $A$ defined by $\pi_{y, \lambda}(a)=$ $\langle\pi(a) y, \lambda\rangle,(a \in A)$. If $\pi$ is continuous, then $\pi_{y, \lambda} \in A^{*}$ for all $y \in Y, \lambda \in Y^{*}$. Occasionally we shall need to consider anti-representations; these are linear maps $\pi: A \longrightarrow \mathscr{L}(Y)$, satisfying $\pi(a b)=\pi(b) \pi(a)$, for all $a, b \in A$. Our definitions of continuity and coordinate functions apply to anti-representations without change.

Given a Banach algebra $A$, we shall assume its dual space $A^{*}$ is equipped with its natural Banach $A$-bimodule structure. Let $X$ be a norm closed $A$-subbimodule of $A^{*}$. Given $\Psi \in X^{*}, \lambda \in X$, we can define $\Psi \cdot \lambda \in A^{*}$ by $\langle\Psi \cdot \lambda, a\rangle=\langle\Psi, \lambda \cdot a\rangle$. If $\Psi \cdot \lambda \in X$ for all choices of $\Psi \in X^{*}$ and $\lambda \in X$, then $X$ is called a topologically left introverted subspace of $A^{*}$. The dual of such a subspace can be made into a Banach algebra if for $\Phi, \Psi \in X^{*}$ we define $\Phi \square \Psi \in X^{*}$ by $\langle\Phi \square \Psi, \lambda\rangle=\langle\Phi, \Psi \cdot \lambda\rangle$. In particular, by taking $X=A^{*}$, we obtain the first (or the left) Arens product on 
$A^{* *}$, defined by Arens in [1,2]. If $X$ is faithful (that is, $a=0$ whenever $\lambda(a)=0$ for all $\lambda \in X)$, then the natural map of $A$ into $X^{*}$ is an embedding, and we will regard $A$ as a subalgebra of $\left(X^{*}, \square\right)$. The space $X^{*}$ can be equipped with the $w^{*}$-topology $\sigma\left(X^{*}, X\right)$. It is well known (and easy to verify) that for each $\Phi \in X^{*}$, the map $\Psi \mapsto \Psi \square \Phi, X^{*} \longrightarrow X^{*}$, is $w^{*}$-continuous.

Right topologically introverted subspaces $X$ of $A^{*}$ are defined similarly; for these spaces, $X^{*}$ can be equipped with the second (or the right) Arens product. A space which is both left and right topologically introverted is called topologically introverted. For more information see for example, Dales-Lau [10].

As an example of a topologically introverted space we mention the space of weakly almost periodic functionals $W A P(A)$, defined as the set of all $\lambda \in A^{*}$ such that the linear map $A \longrightarrow A^{*}, a \mapsto a \cdot \lambda$, is weakly compact. The space of left [right] uniformly continuous functionals on $A$ defined by $L U C(A)=\overline{\operatorname{lin}}\left(A^{*} \cdot A\right)$ $\left[R U C(A)=\overline{\operatorname{lin}}\left(A \cdot A^{*}\right)\right]$ (the closures are in the norm topology) is an example of a left [right] topologically introverted space. For additional examples, see [9, 10, 13, 15, 23, 27.

\section{RePRESEntations of THE DUAL OF INTROVERTED SPACES}

In this paper we shall be mainly interested in representations of Banach algebras on dual Banach spaces, and in particular, on reflexive Banach spaces. The following is the main definition of our paper.

Definition 3.1. Let $\pi: A \longrightarrow \mathscr{L}(Y)$ be a continuous [anti-]representation of a Banach algebra $A$, on a dual Banach space $Y$ with a predual $Y_{*}$. Let $X$ be a normclosed subspace of $A^{*}$. We call $\pi$ subordinate to $X$ if $\pi_{y, \lambda} \in X$, for all $y \in Y, \lambda \in Y_{*}$.

On a dual Banach space $Y$, the $w^{*}$-topology $\sigma\left(Y, Y_{*}\right)$ plays an important role in our investigations. In the definition of subordination, our choice of the coordinate functions $\pi_{y, \lambda}$, with $\lambda \in Y_{*}$ rather than $\lambda \in Y^{*}$, is partly motivated by this fact. Indeed, since $\left(Y, w^{*}\right)^{*}=Y_{*}$ (Dunford-Schwartz [16, Theorem V.3.9]), the elements of $Y_{*}$ are precisely the $w^{*}$-continuous linear functionals on $Y$. For this reason, our notion of subordination might also be called a $w^{*}$-subordination.

Our objective in this section is to establish a bijective correspondence between the continuous representations of $A$ subordinate to $X$ and the normal representations of $X^{*}$, if $X$ is topologically introverted. We start with the following lemma.

Lemma 3.2. Let $Y$ be a Banach space and $\pi: A \longrightarrow \mathscr{L}(Y)$ a continuous representation of $A$ on $Y$. For all $a \in A, f \in Y^{*}$, we have

(i) $a \cdot \pi_{y, f}=\pi_{\pi(a) y, f}$,

(ii) $\pi_{y, f} \cdot a=\pi_{y, \pi(a) * f}$.

Proof. (i) For every $b \in A$ :

$$
\left\langle a \cdot \pi_{y, f}, b\right\rangle=\left\langle\pi_{y, f}, b a\right\rangle=\langle\pi(b a) y, f\rangle=\langle\pi(b)(\pi(a) y), f\rangle=\left\langle\pi_{\pi(a) y, f}, b\right\rangle .
$$

(ii) For every $b \in A$ :

$$
\begin{aligned}
\left\langle\pi_{y, f} \cdot a, b\right\rangle & =\left\langle\pi_{y, f}, a b\right\rangle=\langle\pi(a b) y, f\rangle \\
& =\langle\pi(a)(\pi(b) y), f\rangle=\left\langle\pi(b) y, \pi(a)^{*} f\right\rangle=\left\langle\pi_{y, \pi(a)^{*} f}, b\right\rangle .
\end{aligned}
$$


Next we come to the main result of this section. We remark that various special cases of the result already exist in the literature. For example, statement (i) for the case of $X=W A P(A)$ follows from Runde [33, Theorem 4.10]. The theorem generalizes an earlier result of Filali-Monfared [18, Lemma 2.2] concerning representations of Banach algebras on Hilbert spaces.

Theorem 3.3. Let $A$ be a Banach algebra, $X$ a topologically left (or right) introverted subspace of $A^{*}, Y$ a reflexive Banach space, and $\pi: A \longrightarrow \mathscr{L}(Y)$ a continuous representation subordinate to $X$.

(i) The map

$$
\tilde{\pi}: X^{*} \longrightarrow \mathscr{L}(Y), \quad\langle\widetilde{\pi}(\Psi) y, \lambda\rangle=\left\langle\Psi, \pi_{y, \lambda}\right\rangle,
$$

in which $\Psi \in X^{*}, y \in Y, \lambda \in Y^{*}$, is a normal representation of $X^{*}$ on $Y$. For every $a \in A, \widetilde{\pi}(\dot{a})=\pi(a)$, where $\dot{a}$ is the canonical image of a in $X^{*}$.

(ii) The map $\pi \longrightarrow \widetilde{\pi}$ is a bijection between the set of all (equivalence classes of) continuous representations of $A$ on $Y$ which are subordinate to $X$, and the set of all (equivalence classes of) normal representations of $X^{*}$ on $Y$. Moreover, $\tilde{\pi}$ is topologically irreducible if and only if $\pi$ is topologically irreducible.

Proof. Let us assume that $X$ is topologically left introverted. It follows immediately from the definition that $\widetilde{\pi}$ is linear. To prove that it is a homomorphism, first we show that

$$
\Psi \cdot \pi_{y, \lambda}=\pi_{\widetilde{\pi}(\Psi) y, \lambda} \quad\left(y \in Y, \lambda \in Y^{*}, \Psi \in X^{*}\right) .
$$

In fact, for every $a \in A$ :

$$
\begin{aligned}
\left\langle\Psi \cdot \pi_{y, \lambda}, a\right\rangle & =\left\langle\Psi, \pi_{y, \lambda} \cdot a\right\rangle=\left\langle\Psi, \pi_{y, \pi(a)^{*} \lambda}\right\rangle \\
& =\left\langle\widetilde{\pi}(\Psi)(y), \pi(a)^{*} \lambda\right\rangle \\
& =\langle\pi(a)(\widetilde{\pi}(\Psi) y), \lambda\rangle \\
& =\left\langle\pi_{\widetilde{\pi}(\Psi) y, \lambda}, a\right\rangle
\end{aligned}
$$

where for the second equality we have used Lemma [3.2(ii). Therefore for all $\Psi_{1}, \Psi_{2} \in X^{*}, y \in Y$, and $\lambda \in Y^{*}$, we can write

$$
\begin{aligned}
\left\langle\widetilde{\pi}\left(\Psi_{1} \square \Psi_{2}\right) y, \lambda\right\rangle & =\left\langle\Psi_{1} \square \Psi_{2}, \pi_{y, \lambda}\right\rangle \\
& =\left\langle\Psi_{1}, \Psi_{2} \cdot \pi_{y, \lambda}\right\rangle \\
& =\left\langle\Psi_{1}, \pi_{\tilde{\pi}\left(\Psi_{2}\right) y, \lambda}\right\rangle \\
& =\left\langle\widetilde{\pi}\left(\Psi_{1}\right)\left(\widetilde{\pi}\left(\Psi_{2}\right) y\right), \lambda\right\rangle,
\end{aligned}
$$

which proves that $\widetilde{\pi}\left(\Psi_{1} \square \Psi_{2}\right)=\widetilde{\pi}\left(\Psi_{1}\right) \widetilde{\pi}\left(\Psi_{2}\right)$; that is, $\widetilde{\pi}$ is a homomorphism.

The proofs of the remaining statements of the theorem are essentially straightforward verifications, and are omitted for briefness.

Remarks 3.4. (1) The representation $\widetilde{\pi}$ in Theorem 3.3 can be viewed as the adjoint of the continuous linear map

$$
T: Y \widehat{\otimes} Y^{*} \longrightarrow X, \quad y \otimes \lambda \mapsto \pi_{y, \lambda} .
$$


The adjoint operator $T^{*}: X^{*} \longrightarrow\left(Y \widehat{\otimes} Y^{*}\right)^{*} \cong \mathscr{L}(Y)$ is given by

$$
\left\langle T^{*}(\Psi), y \otimes \lambda\right\rangle=\langle\Psi, T(y \otimes \lambda)\rangle=\left\langle\Psi, \pi_{y, \lambda}\right\rangle, \quad\left(y \in Y, \lambda \in Y^{*}\right),
$$

which implies that $\tilde{\pi}=T^{*}$.

(2) Suppose that $A$ is an Arens regular, involutive Banach algebra. It is wellknown (and easily verified) that the second conjugate-linear adjoint of the involution operator on $A$ gives an involution on $A^{* *}$ (Civin and Yood [8, Theorem 6.2]). If $X \subset A^{*}$ is a topologically introverted subspace such that $f^{*} \in X$ whenever $f \in X,\left(f^{*}(a):=\overline{f\left(a^{*}\right)}\right)$, then $X^{*}=A^{* *} / X^{\circ}$ inherits the involution from $A^{* *}$. If $H$ is a Hilbert space, and $\pi: A \longrightarrow \mathscr{L}(H)$ is an involutive representation of $A$ subordinate to $X$, then $\widetilde{\pi}$ is also an involutive representation of $X^{*}$. A particular case of this situation is when $A$ is a $\mathrm{C}^{*}$-algebra. Theorem 3.3 implies that any involutive representation $\pi$ of $A$ on a Hilbert space has an extension to a normal, involutive representation of $A^{* *}$. This is the well-known normal extension of $\pi$ to the enveloping von Neumann algebra of $A$ (see Dixmier [14, (12.1.5)]).

(3) The representation $\widetilde{\pi}$ may not be faithful even if $\pi$ is faithful. As an example, consider the left regular representation $\pi: L^{1}(G) \longrightarrow \mathscr{L}\left(L^{2}(G)\right), \pi(f) k=f * k$, ( $f \in L^{1}(G), k \in L^{2}(G)$ ). This representation is faithful, since if $\left(k_{\alpha}\right)$ is a bounded approximate identity of $L^{1}(G)$ such that each $k_{\alpha}$ is a continuous function with compact support, then $k_{\alpha} \in L^{2}(G)$ for all $\alpha$, and $\pi(f)=0$ implies that $\pi(f) k_{\alpha}=$ $f * k_{\alpha}=0$ for all $\alpha$, and hence $f=0$. However, the representation $\tilde{\pi}$ of $L^{1}(G)^{* *}$ is not faithful if $G$ is infinite, since $W A P\left(L^{1}(G)\right)$ is a proper closed subspace of $L^{\infty}(G)$ (that is, $L^{1}(G)$ is not Arens regular); see Young [37]. Hence by the HahnBanach theorem there exists a non-zero $\Phi \in L^{1}(G)^{* *}$ such that $\Phi\left(W A P\left(L^{1}(G)\right)\right)=$ $\{0\}$. Since $\pi$ is subordinate to $W A P\left(L^{1}(G)\right)$ (Filali-Monfared [18, Lemma 2.3]), it follows that

$$
\langle\widetilde{\pi}(\Phi) k, h\rangle=\left\langle\Phi, \pi_{k, h}\right\rangle=0,
$$

for all $k, h \in L^{2}(G)$. That is, $\tilde{\pi}(\Phi)=0$.

\section{WEAKLY ALMOST PERIODIC FUNCTIONALS}

Bonsall and Duncan have shown that if $A$ is a unital Banach algebra, then each non-zero $f \in A^{*}$ can be represented as a coordinate function of some dual representation of $A$ (in the sense defined in 4 ); in other words, there exist a Banach $A$-module pairing $(X, Y,\langle\rangle$,$) and cyclic vectors x_{0} \in X$ and $y_{0} \in Y$ such that $f(a)=\left\langle a x_{0}, y_{0}\right\rangle$ for every $a \in A$ (see also Bonsall-Duncan [6, section 29]). In this result, the representation space $X$ is not necessarily reflexive, nor is the representation subordinate to any particular subspace of $A^{*}$.

In this section we shall be interested in representing weakly almost periodic functionals as coordinate functions of continuous representations on reflexive Banach spaces (Theorem 4.4). As we mentioned in the introduction, such representations are always subordinate to $W A P(A)$. Our result strengthens Kaijser's result [26. Lemma 4.12] by replacing the identity of $A$ with a bounded approximate identity.

We should note that our method of the proof of Theorem 4.4 has been inspired by the WAP-representation theorem of Megrelishvili [30, Theorem 4.6], which deals primarily with the representations of topological semigroups (see also the expository paper of Galindo [20, Proposition 1.6]). 
We start with a lemma which will be needed later.

Lemma 4.1. Let $A$ be a Banach algebra and $X \subset A^{*}$ be a faithful, topologically left (or right) introverted subspace of $A^{*}$.

(i) If $A$ has a bounded left approximate identity, then every $\lambda \in X$ is a coordinate function of the continuous representation

$$
L: A \longrightarrow \mathscr{L}(X), \quad L(a) \mu=a \cdot \mu \quad(a \in A, \mu \in X) .
$$

(ii) If $A$ has a bounded right approximate identity, then every $\lambda \in X$ is a coordinate function of the continuous anti-representation

$$
R: A \longrightarrow \mathscr{L}(X), \quad R(a) \mu=\mu \cdot a \quad(a \in A, \mu \in X) .
$$

Proof. (i) The fact that $L$ is a representation and $\|L\| \leq 1$ is straightforward to verify. Let $\left(a_{\alpha}\right)$ be a bounded left approximate identity of $A$, and let $\Phi_{0}$ be any $w^{*}$-cluster point of this net in $X^{*}$. Then $\Phi_{0} \cdot a=a$, for all $a \in A$. Let $\lambda \in X$. Given any $a \in A$, we have

$$
L_{\lambda, \Phi_{0}}(a)=\left\langle L(a) \lambda, \Phi_{0}\right\rangle=\left\langle a \cdot \lambda, \Phi_{0}\right\rangle=\left\langle\lambda, \Phi_{0} \cdot a\right\rangle=\langle\lambda, a\rangle,
$$

which proves that $\lambda=L_{\lambda, \Phi_{0}}$.

(ii) If $\Psi_{0}$ is a $w^{*}$-cluster point in $X^{*}$ of a bounded right approximate identity of $A$, then $a \cdot \Psi_{0}=a$, for all $a \in A$, and by a similar argument to that given in (i), $\lambda=R_{\lambda, \Psi_{0}}$.

Remark 4.2. The above lemma shows that the left regular representation $L$ of a Banach algebra $A$ on its dual space $A^{*}$ is not subordinate to any proper subspace of $A^{*}$.

Let $W$ be a convex, symmetric, and bounded subset of a Banach space $(X,\|\cdot\|)$, and let $B_{X}$ be the closed unit ball of $X$. For each $n=1,2, \ldots$, the gauge $\|\cdot\|_{n}$ of the set $U_{n}=2^{n} W+2^{-n} B_{X}$, defined by $\|x\|_{n}=\inf \left\{t \geq 0: x \in t U_{n}\right\}$, is a norm on $X$ equivalent to $\|\cdot\|$. For each $x \in X$, we define $\|x\|^{\prime}=\left(\sum_{n=1}^{\infty}\|x\|_{n}^{2}\right)^{1 / 2}$ and we let $Y=\left\{x \in X:\|x\|^{\prime}<\infty\right\}$ and $B_{Y}=\left\{x \in Y:\|x\|^{\prime} \leq 1\right\}$. We let $j: Y \rightarrow X$ be the natural embedding. The following result, which shall be needed for our next theorem, is due to Davis, Figiel, Johnson, and Pełczyński [11, Lemma 1].

Lemma 4.3 (Davis et al). Under the above conditions,

(i) $W \subset B_{Y}$;

(ii) $\left(Y,\|\cdot\|^{\prime}\right)$ is a Banach space and $\|j\| \leq 1$;

(iii) $j^{* *}: Y^{* *} \longrightarrow X^{* *}$ is injective and $\left(j^{* *}\right)^{-1}(X)=Y$;

(iv) $Y$ is reflexive if and only if $W$ is weakly relatively compact.

Let $\lambda \in W A P(A)$. Using the above notation, let $X=A^{*}$, and let $W$ be the norm closure of $\{a \cdot \lambda: a \in A,\|a\| \leq 1\}$ in $A^{*}$. Then $W$ is a convex, bounded, and weakly compact subset of $A^{*}$. Hence if $Y$ is as in Lemma 4.3, then $Y$ is a reflexive Banach space, $Y \subset A^{*}$, the natural inclusion $j: Y \longrightarrow A^{*}$ is continuous and $\|j\| \leq 1$, and moreover, $W \subset B_{Y}$. Now we can state the main result of this section.

Theorem 4.4. Let $A$ be a Banach algebra with a bounded two-sided approximate identity and let $0 \neq \lambda \in W A P(A)$. Let $L: A \longrightarrow \mathscr{L}\left(A^{*}\right)$ be defined by $L(a) \mu=$ $a \cdot \mu,\left(a \in A, \mu \in A^{*}\right)$. Then the map

$$
\pi: A \longrightarrow \mathscr{L}(Y), \quad \pi(a)=L(a) \circ j=\left.L(a)\right|_{Y},
$$


is a continuous representation of $A$ on the reflexive Banach space $Y$, and $\lambda$ is a coordinate function of $\pi$. Moreover, $\pi$ is faithful if $Y$ separates the points of $A$.

Proof. Let $a \in A$ and $\mu \in Y$. If $t \geq 0$ is such that $\mu \in t U_{n}$, then $\mu \in 2^{n} t W+$ $2^{-n} t B_{A^{*}}$, and therefore

$$
a \cdot \mu \in 2^{n} t a \cdot W+2^{-n} t a \cdot B_{A^{*}} \subset 2^{n}\|a\| t W+2^{-n}\|a\| t B_{A^{*}} .
$$

It follows that $\|a \cdot \mu\|_{n} \leq\|a\| t$, and hence

$$
\|a \cdot \mu\|_{n} \leq\|a\|\|\mu\|_{n} .
$$

Thus for every $a \in A$ and $\mu \in Y$, we have

$$
\|a \cdot \mu\|^{\prime}=\left(\sum_{n=1}^{\infty}\|a \cdot \mu\|_{n}^{2}\right)^{1 / 2} \leq\|a\|\left(\sum_{n=1}^{\infty}\|\mu\|_{n}^{2}\right)^{1 / 2}=\|a\|\|\mu\|^{\prime} .
$$

Hence $L(a) \mu \in Y$, and $\left.L(a)\right|_{Y}: Y \longrightarrow Y$ is a continuous linear operator and $\left\|\left.L(a)\right|_{Y}\right\| \leq\|a\|$. Thus $\|\pi\| \leq 1$. The fact that $\pi$ is a representation is now immediate.

Let $\left(e_{\alpha}\right)$ be an approximate identity for $A$ bounded by $r>0$. Then it is easily verified that $e_{\alpha} \cdot \lambda \rightarrow \lambda$ in $w^{*}$-topology of $A^{*}$. Since $\lambda \in W A P(A)$, for a suitable subnet of $\left(e_{\alpha}\right)$ we must also have $e_{\alpha_{\beta}} \cdot \lambda \rightarrow \lambda^{\prime} \in A^{*}$ in the weak topology. Since $\left\{a \cdot \lambda: a \in B_{A}(0, r)\right\}$ is convex, and the norm and weak closures of convex sets are equal (cf. [16, Theorem V.3.13]), we have

$$
\lambda=\lambda^{\prime} \in\left\{a \cdot \lambda: a \in B_{A}(0, r)\right\}^{-\|\cdot\|}=r W \subset Y .
$$

Let $\Phi_{0}$ be a $w^{*}$-cluster point of $\left(e_{\alpha}\right)$ in $A^{* *}$. This is a mixed identity for $A^{* *}$, and in particular, $\Phi_{0} \cdot a=a$, for each $a \in A$. Let $\Phi_{00}=\left.\Phi_{0}\right|_{Y}$. Then $\Phi_{00} \in Y^{*}$ since for every $\mu \in Y$ :

$$
\left|\Phi_{00}(\mu)\right|=\left|\Phi_{0}(\mu)\right| \leq\left\|\Phi_{0}\right\|_{A^{* *}}\|\mu\|_{A^{*}} \leq\left\|\Phi_{0}\right\|_{A^{* *}}\|j\|\|\mu\|^{\prime} .
$$

In addition, if $a \in A$, then we have

$$
\pi_{\lambda, \Phi_{00}}(a)=\left\langle\pi(a) \lambda, \Phi_{00}\right\rangle=\left\langle a \cdot \lambda, \Phi_{0}\right\rangle=\left\langle\lambda, \Phi_{0} \cdot a\right\rangle=\langle\lambda, a\rangle,
$$

which proves that $\pi_{\lambda, \Phi_{00}}=\lambda$.

Finally suppose that $Y$ separates the points of $A$ and for some $a_{0} \in A, \pi\left(a_{0}\right)=0$. Then $a_{0} \cdot y=0$ for all $y \in Y$. Therefore $\left\langle y, a a_{0}\right\rangle=0$ for all $a \in A$, and since $A$ has a bounded approximate identity, it follows that $\left\langle y, a_{0}\right\rangle=0$ for all $y \in Y$. Thus $a_{0}=0$ and $\pi$ is faithful.

Remark 4.5. For almost periodic functions on locally compact groups, the following result is known: $f \in A P\left(L^{1}(G)\right)=A P(G)$ if and only if $f$ is a uniform limit of coordinate functions of continuous representations $\pi_{k}: G \longrightarrow \mathcal{U}\left(H_{k}\right)$, where each $H_{k}$ is a finite-dimensional Hilbert space and $\mathcal{U}\left(H_{k}\right)$ is the group of unitary operators on $H_{k}$ (see Galindo [20, Proposition 1.6(6)]). It follows from FilaliMonfared [18, Lemma 2.3] that a uniform limit of coordinate functions of finitedimensional continuous representations of a Banach algebra $A$ is in $A P(A)$.

The paper by Daws [12] is a study of the representations of dual Banach algebras on reflexive Banach spaces. Let us recall that a dual Banach algebra is a Banach algebra $A$ such that there exists a Banach space $A_{*}$ with the properties that $A=$ $\left(A_{*}\right)^{*}$ and the multiplication on $A$ is separately $w^{*}$-continuous (cf. Runde [32]). 
The space $W A P(A)^{*}$ is an example of a dual Banach algebra (Runde 33, Theorem 4.10]).

Our main objective in what follows is to derive the following result of Daws [12, Corollary 3.8] from our Theorem 4.4. We should mention that our proof does not use the technique of interpolation spaces (Beauzamy [3]). Our idea is based on the fact that a dual Banach algebra embeds isometrically into $W A P(A)^{*}$ (Runde [33. Corollary 4.6]), and hence one can reduce the problem to finding an isometric embedding of $W A P(A)^{*}$.

Theorem 4.6 (Daws). Every dual Banach algebra is isometrically isomorphic to a closed subalgebra of $\mathscr{L}(Y)$, for some reflexive Banach space $Y$.

Proof. Let $A$ be a dual Banach algebra with a predual $A_{*}$. Since the unitization of a dual Banach algebra remains a dual Banach algebra, we may assume with no loss of generality that $A$ is unital.

For each $\lambda \in W A P(A)$, let $\pi^{\lambda}: A \longrightarrow \mathscr{L}\left(Y_{\lambda}\right)$ be a representation of $A$ on a reflexive Banach space $Y_{\lambda}$ such that $\left\|\pi^{\lambda}\right\| \leq 1$ and $\lambda$ is a coordinate function of $\pi^{\lambda}$ (Theorem 4.4). We define

$$
T_{\lambda}: Y_{\lambda} \widehat{\otimes} Y_{\lambda}^{*} \longrightarrow W A P(A), \quad y_{\lambda} \otimes \phi_{\lambda} \mapsto \pi_{y_{\lambda}, \phi_{\lambda}}^{\lambda},
$$

so that $T_{\lambda}$ is a continuous linear map with $\left\|T_{\lambda}\right\| \leq 1$. Let $Y=\ell^{2}-\oplus_{\lambda} Y_{\lambda}$ be the $\ell^{2}$-direct sum of the $Y_{\lambda}$, with $\lambda \in W A P(A)$. Then $Y$ is a reflexive Banach space and $Y^{*}=\ell^{2}-\oplus_{\lambda} Y_{\lambda}^{*}$. Let

$$
\begin{gathered}
T: Y \widehat{\otimes} Y^{*} \longrightarrow W A P(A), \\
\left(y_{\lambda}\right) \otimes\left(\phi_{\lambda}\right) \mapsto \sum_{\lambda} T_{\lambda}\left(y_{\lambda} \otimes \phi_{\lambda}\right)=\sum_{\lambda} \pi_{y_{\lambda}, \phi_{\lambda}}^{\lambda} .
\end{gathered}
$$

Then $T$ is a continuous linear map with $\|T\| \leq 1$. By Theorem 4.4, the map $T$ is surjective. We claim that the adjoint map

$$
T^{*}: W A P(A)^{*} \longrightarrow\left(Y \widehat{\otimes} Y^{*}\right)^{*} \cong \mathscr{L}(Y)
$$

is an isometric algebra homomorphism. To prove this claim, first we show that given $\nu \in W A P(A)^{*}, y=\left(y_{\lambda}\right) \in Y$, and $\phi=\left(\phi_{\lambda}\right) \in Y^{*}$, we have

$$
\begin{aligned}
\left(T^{*} \nu\right) y & =\left(\left(T_{\lambda}^{*} \nu\right) y_{\lambda}\right)_{\lambda} \in Y, \\
\sum_{\lambda} \nu \cdot \pi_{y_{\lambda}, \phi_{\lambda}}^{\lambda} & =T\left(\left(T^{*} \nu\right) y \otimes \phi\right) \in W A P(A) .
\end{aligned}
$$

In fact,

$$
\begin{aligned}
\left\langle\left(T^{*} \nu\right) y, \phi\right\rangle & =\left\langle T^{*} \nu, y \otimes \phi\right\rangle=\left\langle\nu, \sum_{\lambda} T_{\lambda}\left(y_{\lambda} \otimes \phi_{\lambda}\right)\right\rangle=\sum_{\lambda}\left\langle T_{\lambda}^{*} \nu, y_{\lambda} \otimes \phi_{\lambda}\right\rangle \\
& =\sum_{\lambda}\left\langle\left(T_{\lambda}^{*} \nu\right) y_{\lambda}, \phi_{\lambda}\right\rangle=\left\langle\left(\left(T_{\lambda}^{*} \nu\right) y_{\lambda}\right),\left(\phi_{\lambda}\right)\right\rangle
\end{aligned}
$$


which proves (6). To prove (7), we note that for every $a \in A$, we have

$$
\begin{aligned}
\left\langle\sum_{\lambda} \nu \cdot \pi_{y_{\lambda}, \phi_{\lambda}}^{\lambda}, a\right\rangle & =\sum_{\lambda}\left\langle\nu, \pi_{y_{\lambda}, \phi_{\lambda}}^{\lambda} \cdot a\right\rangle \\
(\text { Lemma } 3.2(\mathrm{ii})) & =\sum_{\lambda}\left\langle\nu, \pi_{y_{\lambda}, \pi^{\lambda}(a)^{*} \phi_{\lambda}}^{\lambda}\right\rangle \\
& =\left\langle\nu, T\left(y \otimes\left(\pi^{\lambda}(a)^{*} \phi_{\lambda}\right)\right)\right\rangle \\
& =\left\langle\left(T^{*} \nu\right) y,\left(\pi^{\lambda}(a)^{*} \phi_{\lambda}\right)\right\rangle \\
(\text { by (6) })) & =\left\langle\left(\left(T_{\lambda}^{*} \nu\right) y_{\lambda}\right),\left(\pi^{\lambda}(a)^{*} \phi_{\lambda}\right)\right\rangle \\
& =\sum_{\lambda}\left\langle\pi^{\lambda}(a)\left(T_{\lambda}^{*} \nu\right) y_{\lambda}, \phi_{\lambda}\right\rangle \\
& =\left\langle\sum_{\lambda} \pi_{\left(T_{\lambda}^{*} \nu\right) y_{\lambda}, \phi_{\lambda}}^{\lambda}, a\right\rangle \\
& =\left\langle T\left(\left(\left(T_{\lambda}^{*} \nu\right) y_{\lambda}\right) \otimes\left(\phi_{\lambda}\right)\right), a\right\rangle \\
\text { (by (6) }) & =\left\langle T\left(\left(T^{*} \nu\right) y \otimes \phi\right), a\right\rangle,
\end{aligned}
$$

which completes the proof of (7). Now we can verify $T^{*}(\mu \square \nu)=\left(T^{*} \mu\right)\left(T^{*} \nu\right)$, for all $\mu, \nu \in W A P(A)^{*}$. In fact, for all $y \in Y$ and $\phi \in Y^{*}$, we have

$$
\begin{aligned}
\left\langle T^{*}(\mu \square \nu) y, \phi\right\rangle & =\left\langle T^{*}(\mu \square \nu), y \otimes \phi\right\rangle \\
& =\langle\mu \square \nu, T(y \otimes \phi)\rangle \\
& =\left\langle\mu \square \nu, \sum_{\lambda} \pi_{y_{\lambda}, \phi_{\lambda}}^{\lambda}\right\rangle \\
& =\left\langle\mu, \sum_{\lambda} \nu \cdot \pi_{y_{\lambda}, \phi_{\lambda}}^{\lambda}\right\rangle \\
(\text { by (7)) } & =\left\langle\mu, T\left(\left(T^{*} \nu\right) y \otimes \phi\right)\right\rangle \\
& =\left\langle T^{*} \mu,\left(T^{*} \nu\right) y \otimes \phi\right\rangle \\
& =\left\langle\left(T^{*} \mu\right)\left(T^{*} \nu\right) y, \phi\right\rangle,
\end{aligned}
$$

which proves that $T^{*}(\mu \square \nu)=\left(T^{*} \mu\right)\left(T^{*} \nu\right)$.

Next, we check that $T^{*}$ is an isometry. Since $\left\|T^{*}\right\| \leq 1$, it follows that $\left\|T^{*} \mu\right\| \leq$ $\|\mu\|$ for every $\mu \in W A P(A)^{*}$. Let $e$ be the identity of $A$ and let $j_{\lambda}: Y_{\lambda} \longrightarrow A^{*}$ be the natural inclusion map (see the discussion following Lemma 4.3). Let $e_{\lambda}=j_{\lambda}^{*}(e)$, so that $e_{\lambda}$ is the restriction of $e$ to $Y_{\lambda}$, when $e$ is viewed as an element of $A^{* *}$. Since $\left\|j_{\lambda}\right\| \leq 1$, it follows that $\left\|e_{\lambda}\right\| \leq 1$. Let $\epsilon>0$, and pick $\lambda \in W A P(A)$ such that $\|\lambda\| \leq 1$ and $|\langle\mu, \lambda\rangle| \geq\|\mu\|-\epsilon$. Then

$$
T_{\lambda}\left(\lambda \otimes e_{\lambda}\right)=\pi_{\lambda, e_{\lambda}}^{\lambda}=\lambda,
$$

and thus

$$
\begin{aligned}
\left\|T^{*} \mu\right\| \geq\left\|T_{\lambda}^{*} \mu\right\| \geq\left|\left\langle T_{\lambda}^{*} \mu, \lambda \otimes e_{\lambda}\right\rangle\right| & =\left|\left\langle\mu, T_{\lambda}\left(\lambda \otimes e_{\lambda}\right)\right\rangle\right| \\
& =|\langle\mu, \lambda\rangle| \geq\|\mu\|-\epsilon .
\end{aligned}
$$

Since $\epsilon>0$ was arbitrary, we have shown that $\left\|T^{*} \mu\right\| \geq\|\mu\|$, which leads to our claim that $T^{*}$ is an isometry. 
Therefore, $W A P(A)^{*}$ is isometrically embedded into $\mathscr{L}(Y)$. Now as noted in Runde [33, Remark 3, p. 141], since $A$ is a dual Banach algebra, it embeds isometrically into $W A P(A)^{*}$ by Runde [33, Corollary 4.6], thereby completing the proof of the theorem.

As Daws communicated to us, Theorem 4.4 can also be deduced from his results on the representations of dual Banach algebras in [12. This is obtained with the help of Theorem 3.3(ii). Let $A$ be a Banach algebra with a bounded approximate identity and let $\lambda \in W A P(A)$. Since the natural image of the mixed identity of $A^{* *}$ in $W A P(A)^{*}$ is the identity of $W A P(A)^{*}, W A P(A)^{*}$ is a unital dual Banach algebra. By Daws [12, Lemma 3.4 and Theorem 3.6], there exists a normal representation $\sigma$ of $W A P(A)^{*}$ on a reflexive Banach space $Y$ such that $\lambda$ is a coordinate function of $\sigma$. By Theorem 3.3(ii), we can write $\sigma=\tilde{\pi}$, for some continuous representation $\pi: A \longrightarrow \mathscr{L}(Y)$. Let $y \in Y, f \in Y^{*}$ be such that $\tilde{\pi}_{y, f}=\lambda$. Then for each $\Phi \in W A P(A)^{*}$,

$$
\langle\lambda, \Phi\rangle=\left\langle\widetilde{\pi}_{y, f}, \Phi\right\rangle=\langle\widetilde{\pi}(\Phi) y, f\rangle=\left\langle\pi_{y, f}, \Phi\right\rangle ;
$$

that is, $\lambda=\pi_{y, f}$, which is what we wanted to show.

\section{LUC AND RUC-REPRESENTATION THEOREMS}

Let $G$ be a locally compact group. We recall that the space of left uniformly continuous functions on $G$, denoted by $\operatorname{LUC}(G)$, consists of all bounded continuous functions $f$ on $G$ for which the left translation map $G \longrightarrow \mathscr{C}^{b}(G), a \mapsto{ }_{a} f$, is continuous. The space $R U C(G)$ is defined similarly. These spaces are special cases of the spaces $L U C(A)$ and $R U C(A)$ (section 2); in fact, Lau 28] has shown that for a locally compact group $G$, we have $L U C\left(L^{1}(G)\right)=L U C(G)$, and $R U C\left(L^{1}(G)\right)=$ $R U C(G)$.

In this section we shall consider representations that are subordinate to the space $L U C(G)$, or more generally, to $L U C(A)$. (For a recent study of unitary representations whose coordinate functions vanish at infinity, see Ghandehari 22]). In Theorem 5.2 we show that to every continuous unitary representation of a locally compact group $G$ on a Hilbert space $H$ (cf. Hewitt-Ross [24, (22.9), (22.20)]), one can associate a conjugate [anti-]representation $\left[\pi^{\prime}\right] \pi$ of $L^{1}(G)$ on $\mathscr{L}(H)$, which is subordinate to $[R U C(G)] \operatorname{LUC}(G)$. The converse of this result will be proved in Theorem 5.3 .

The following result was proved for the left regular representation of $G$ on $L^{2}(G)$ by Størmer 35] when $G$ is abelian, and by Ghahramani 21 for general locally compact groups. Both Størmer and Ghahramani showed that the associated representation to the left regular representation is an isometry. This is of course not true in general.

Lemma 5.1. Let $V: G \longrightarrow \mathscr{L}(H)$ be a continuous unitary representation of $G$.

(i) The map $\pi: M(G) \longrightarrow \mathscr{L}(\mathscr{L}(H))$, defined by

$$
\pi(\mu) T=\int_{G} V(t) T V(t)^{*} d \mu(t) \quad(\mu \in M(G), T \in \mathscr{L}(H))
$$

is a continuous representation with $\|\pi\| \leq 1$. 
(ii) The map $\pi^{\prime}: M(G) \longrightarrow \mathscr{L}(\mathscr{L}(H))$, defined by

$$
\pi^{\prime}(\mu) T=\int_{G} V(t)^{*} T V(t) d \mu(t) \quad(\mu \in M(G), T \in \mathscr{L}(H)),
$$

is a continuous anti-representation with $\left\|\pi^{\prime}\right\| \leq 1$.

The integrals appearing in (8) and (9) are operator-valued, and the identity (8) (and those similar to it) is to be interpreted as

$$
\langle\pi(\mu) T x \mid y\rangle=\int_{G}\left\langle V(t) T V(t)^{*} x \mid y\right\rangle d \mu(t) \quad(x, y \in H) .
$$

For a brief review of these integrals see Folland [19, Appendix 3], and for a more detailed treatment, Bourbaki [7, Section VI.1.3].

Proof. Let $\mu, \nu \in M(G)$ and $T \in \mathscr{L}(H)$. Then

$$
\begin{aligned}
\pi(\mu * \nu) T & =\int_{G} V(t) T V(t)^{*} d \mu * \nu(t) \\
& =\int_{G} \int_{G} V\left(g g^{\prime}\right) T V\left(g g^{\prime}\right)^{*} d \nu\left(g^{\prime}\right) d \mu(g) \\
& =\int_{G} \int_{G} V(g) V\left(g^{\prime}\right) T V\left(g^{\prime}\right)^{*} V(g)^{*} d \nu\left(g^{\prime}\right) d \mu(g) \\
& =\int_{G} V(g)\left(\int_{G} V\left(g^{\prime}\right) T V\left(g^{\prime}\right)^{*} d \nu\left(g^{\prime}\right)\right) V(g)^{*} d \mu(g) \\
& =\int_{G} V(g)(\pi(\nu) T) V(g)^{*} d \mu(g) \\
& =\pi(\mu)(\pi(\nu) T) .
\end{aligned}
$$

Thus $\pi(\mu * \nu)=\pi(\mu) \pi(\nu)$.

For each $\mu \in M(G)$ :

$$
\begin{aligned}
\|\pi(\mu)\| & =\sup _{\|T\| \leq 1}\|\pi(\mu) T\|=\sup _{\|T\| \leq 1}\left\|\int_{G} V(t) T V(t)^{*} d \mu(t)\right\| \\
& \leq \sup _{\|T\| \leq 1} \int_{G}\left\|V(t) T V(t)^{*}\right\| d|\mu|(t) \\
& \leq \sup _{\|T\| \leq 1}\|T\||\mu|(G)=\|\mu\| .
\end{aligned}
$$

That is, $\|\pi\| \leq 1$.

The proof of (ii) is similar.

In the following, we shall call $\pi$ and $\pi^{\prime}$ given in (8) and (9) and their restrictions to $L^{1}(G)$, the conjugate representation and anti-representation associated with $V$. We recall that the predual of $\mathscr{L}(H)$ is the space $\mathscr{L}(H)_{*} \cong H \widehat{\otimes} H$, where $\widehat{\otimes}$ denotes the project tensor product. 
Theorem 5.2. Let $V: G \longrightarrow \mathscr{L}(H)$ be a continuous unitary representation, and $\left[\pi^{\prime}\right] \pi$ be the associated conjugate [anti-]representation of $L^{1}(G)$.

(i) If $T \in \mathscr{L}(H)$ and $T_{*}=\sum_{i=1}^{\infty} x_{i} \otimes y_{i} \in \mathscr{L}(H)_{*}$, then for locally almost all $t \in G$ :

$$
\begin{aligned}
& \pi_{T, T_{*}}(t)=\sum_{i=1}^{\infty}\left\langle T V(t)^{*} x_{i} \mid V(t)^{*} y_{i}\right\rangle, \\
& \pi_{T, T_{*}}^{\prime}(t)=\sum_{i=1}^{\infty}\left\langle T V(t) x_{i} \mid V(t) y_{i}\right\rangle .
\end{aligned}
$$

(ii) $\pi$ and $\pi^{\prime}$ are subordinate to $L U C(G)$ and $\operatorname{RUC}(G)$, respectively.

Proof. We give the proof for $\pi$ and leave the verification of the statements for $\pi^{\prime}$ to the reader.

(i) Since $\pi$ is continuous, it follows that $\pi_{T, T_{*}} \in L^{\infty}(G)$. Let $h \in L^{1}(G)$; then we have

$$
\begin{aligned}
\pi_{T, T_{*}}(h) & =\left\langle\pi(h) T, T_{*}\right\rangle=\sum_{i=1}^{\infty}\left\langle\pi(h) T, x_{i} \otimes y_{i}\right\rangle=\sum_{i=1}^{\infty}\left\langle\pi(h) T x_{i} \mid y_{i}\right\rangle \\
& =\sum_{i=1}^{\infty}\left\langle\int_{G} V(t) T V(t)^{*} x_{i} h(t) d t \mid y_{i}\right\rangle \\
& =\int_{G} \sum_{i=1}^{\infty}\left\langle V(t) T V(t)^{*} x_{i} \mid y_{i}\right\rangle h(t) d t,
\end{aligned}
$$

from which (i) follows.

(ii) For each $t \in G$, let us define $f_{i}(t)=\left\langle T V(t)^{*} x_{i} \mid V(t)^{*} y_{i}\right\rangle$. Then $f_{i} \in \mathscr{C}^{b}(G)$ and

$$
\sum_{i=1}^{\infty}\left\|f_{i}\right\| \leq\|T\| \sum_{i=1}^{\infty}\left\|x_{i}\right\|\left\|y_{i}\right\|<\infty,
$$

and hence $\sum_{i} f_{i}$ is uniformly and absolutely convergent in $\mathscr{C}^{b}(G)$. So if we show that $f_{i} \in L U C(G)$, it will follow that $\pi_{T, T_{*}} \in L U C(G)$.

Let $x, y \in H$ and define $f(x)=\left\langle T V(t)^{*} x \mid V(t)^{*} y\right\rangle$. If $\left(s_{\alpha}\right)$ is a net in $G$ and $s_{\alpha} \rightarrow e$, then we need to show that $\left\|_{s_{\alpha}} f-f\right\| \rightarrow 0$. We have

$$
\begin{gathered}
\left\|s_{s_{\alpha}} f-f\right\|=\sup _{t \in G}\left|\left\langle T V\left(s_{\alpha} t\right)^{*} x \mid V\left(s_{\alpha} t\right)^{*} y\right\rangle-\left\langle T V(t)^{*} x \mid V(t)^{*} y\right\rangle\right| \\
=\sup _{t \in G} \mid\left\langle T V\left(s_{\alpha} t\right)^{*} x \mid V\left(s_{\alpha} t\right)^{*} y\right\rangle-\left\langle T V(t)^{*} x \mid V\left(s_{\alpha} t\right)^{*} y\right\rangle \\
\quad+\left\langle T V(t)^{*} x \mid V\left(s_{\alpha} t\right)^{*} y\right\rangle-\left\langle T V(t)^{*} x \mid V(t)^{*} y\right\rangle \mid \\
\leq \sup _{t \in G}\left\|T\left(V\left(s_{\alpha} t\right)^{*}-V(t)^{*}\right) x\right\|\left\|V\left(s_{\alpha} t\right)^{*} y\right\| \\
\quad+\sup _{t \in G}\left\|T V(t)^{*} x\right\|\left\|\left(V\left(s_{\alpha} t\right)^{*}-V(t)^{*}\right) y\right\| \\
\leq\|T\| \sup _{t \in G}\left\|V(t)^{*}\left(V\left(s_{\alpha}\right)^{*} x-x\right)\right\|\left\|V(t)^{*}\left(V\left(s_{\alpha}\right)^{*} y\right)\right\| \\
\quad+\|T\| \sup _{t \in G}\left\|V(t)^{*} x\right\|\left\|V(t)^{*}\left(V\left(s_{\alpha}\right)^{*} y-y\right)\right\| \\
=\|T\|\left\|V\left(s_{\alpha}\right)^{*} x-x\right\|\|y\|+\|T\|\|x\|\left\|V\left(s_{\alpha}\right)^{*} y-y\right\| .
\end{gathered}
$$


Since $V$ is continuous, it follows that $\left\|V\left(s_{\alpha}\right)^{*} x-x\right\| \rightarrow 0$ and $\left\|V\left(s_{\alpha}\right)^{*} y-y\right\| \rightarrow 0$, hence proving that $\left\|_{s_{\alpha}} f-f\right\| \rightarrow 0$.

Next we prove a converse of Theorem 5.2

Theorem 5.3. Let $G$ be a locally compact group. Then every function in $[R U C(G)]$ $L U C(G)$ is a coordinate function of the conjugate [anti-] representation of $L^{1}(G)$ on $\mathscr{L}\left(L^{2}(G)\right)$, associated with the left regular representation of $G$, as defined in (8) and (9).

Proof. First let $f \in L U C(G)$. Using the identity $L U C(G)=L^{1}(G) * L^{\infty}(G)$ (Hewitt-Ross [25, (32.45)]), we can set $f=h * g$, where $h \in L^{1}(G), g \in L^{\infty}(G)$. Let us define $\phi, \psi \in L^{2}(G)$ by $\psi(x)=|h(x)|^{1 / 2},(x \in G)$, and $\phi(x)=0$ if $h(x)=0$, while $\phi(x)=h(x) /|h(x)|^{1 / 2}$ if $h(x) \neq 0$. Then $h=\phi \psi$. Set $T_{*}=\phi \otimes \psi \in L^{2}(G) \widehat{\otimes} L^{2}(G)=$ $\mathscr{L}\left(L^{2}(G)\right)_{*}$, and let $M_{\check{g}} \in \mathscr{L}\left(L^{2}(G)\right)$ be the multiplication operator by $\check{g}$, where $\check{g}(x)=g\left(x^{-1}\right)$. Let $V$ be the left regular representation of $G$ on $L^{2}(G)$ and let

$$
\pi: L^{1}(G) \longrightarrow \mathscr{L}\left(\mathscr{L}\left(L^{2}(G)\right)\right), \quad \pi(k) T=\int_{G} V(t) T V(t)^{*} k(t) d t,
$$

$k \in L^{1}(G), T \in \mathscr{L}\left(L^{2}(G)\right)$, be the conjugate representation associated to $V$. We claim that $f$ is a coordinate function of $\pi$ corresponding to $M_{\breve{g}} \in \mathscr{L}\left(L^{2}(G)\right)$ and $T_{*} \in \mathscr{L}\left(L^{2}(G)\right)_{*} ;$ in other words,

$$
\pi_{M_{\check{g}}, T_{*}}=f .
$$

For every $k \in L^{1}(G)$, we have

$$
\begin{aligned}
\left\langle\pi_{M_{\check{g}}, T_{*}}, k\right\rangle & =\left\langle\pi(k) M_{\check{g}}, \phi \otimes \psi\right\rangle \\
& =\left\langle\left(\pi(k) M_{\check{g}}\right) \phi \mid \psi\right\rangle \\
& =\left\langle\int_{G}\left(V(t) M_{\check{g}} V(t)^{*} \phi\right) k(t) d t \mid \psi\right\rangle \\
& =\int_{G}\left\langle V(t) M_{\check{g}} V(t)^{*} \phi \mid \psi\right\rangle k(t) d t .
\end{aligned}
$$

Therefore, to prove (12), it remains to show that for almost all $t \in G$,

$$
\left\langle V(t) M_{\check{g}} V(t)^{*} \phi \mid \psi\right\rangle=f(t) .
$$

Given $t \in G$, we can write

$$
\begin{aligned}
\left\langle V(t) M_{\check{g}} V(t)^{*} \phi \mid \psi\right\rangle & =\int_{G}\left(V(t) M_{\check{g}} V(t)^{*} \phi\right)(s) \psi(s) d s \\
& =\int_{G}\left(M_{\check{g}} V(t)^{*} \phi\right)\left(t^{-1} s\right) \psi(s) d s \\
& =\int_{G} \check{g}\left(t^{-1} s\right)\left(V(t)^{*} \phi\right)\left(t^{-1} s\right) \psi(s) d s \\
& =\int_{G} g\left(s^{-1} t\right) \phi(s) \psi(s) d s \\
& =\int_{G} g\left(s^{-1} t\right) h(s) d s \\
& =(h * g)(t) \\
& =f(t),
\end{aligned}
$$

which proves (13), and hence (12). 
Finally, if we take $f=g * \check{h} \in R U C(G)=L^{\infty}(G) * L^{1}(G)$, then we can choose $\phi, \psi$, and $T_{*}$, as before, and let

$$
\pi^{\prime}: L^{1}(G) \longrightarrow \mathscr{L}\left(\mathscr{L}\left(L^{2}(G)\right)\right), \quad \pi^{\prime}(k) T=\int_{G} V(t)^{*} T V(t) k(t) d t,
$$

be the conjugate anti-representation associated to $V$. Then, by an argument similar to the one given above, one can show that $\pi_{M_{g}, T_{*}}^{\prime}=f$, as required.

As kindly pointed out by the referee, it would be interesting to know if Theorems 5.2 and 5.3 can be extended to $L U C(G)$ or $R U C(G)$ for a general topological group G. In this regard see the recent study of Lau and Ludwig [29].

Next we turn our attention to the space of left uniformly continuous functionals $L U C(A)$. We shall present a class of representations of $A$ that are subordinate to $L U C(A)$, and moreover, we shall show that every element in $L U C(A)$ is a coordinate function of one such representation.

Theorem 5.4. Let $A$ be a Banach algebra with a bounded right approximate identity. Let $\theta: A \longrightarrow \mathscr{L}(A)$ be a continuous, non-degenerate, anti-representation. Then each of the two maps

(i) $\pi^{\theta}: A \longrightarrow \mathscr{L}\left(\mathscr{L}\left(A, A^{*}\right)\right), \quad \pi^{\theta}(a) T=T \circ \theta(a)$,

(ii) $\check{\pi}^{\theta}: A \longrightarrow \mathscr{L}\left(\mathscr{L}\left(A, A^{*}\right)\right), \quad \check{\pi}^{\theta}(a) T=\theta(a)^{*} \circ T$,

where $a \in A, T \in \mathscr{L}\left(A, A^{*}\right)$, is a continuous representation of $A$ subordinate to $\operatorname{LUC}(A)$.

Proof. We give the proof for $\pi^{\theta}$; the proof for $\check{\pi}^{\theta}$ is similar. Clearly $\pi^{\theta}$ is a linear map; moreover, for all $a, b \in A$,

$$
\pi^{\theta}(a b) T=T \circ \theta(a b)=T \circ(\theta(b) \circ \theta(a))=(T \circ \theta(b)) \circ \theta(a)=\pi^{\theta}(a)\left(\pi^{\theta}(b) T\right),
$$

which shows that $\pi^{\theta}$ is a homomorphism; in addition,

$$
\left\|\pi^{\theta}(a) T\right\|=\|T \circ \theta(a)\| \leq\|\theta\|\|T\|\|a\|,
$$

and hence $\left\|\pi^{\theta}\right\| \leq\|\theta\|$, that is, $\pi^{\theta}$ is a continuous representation.

It remains to show that $\pi^{\theta}$ is subordinate to $\operatorname{LUC}(A)$. Let $T_{*}=a_{1} \otimes a_{2} \in$ $A \widehat{\otimes} A=\mathscr{L}\left(A, A^{*}\right)_{*}$, and let $T \in \mathscr{L}\left(A, A^{*}\right)$. Then for all $c \in A$, we have

$$
\pi_{T, T_{*}}^{\theta}(c)=\left\langle\pi^{\theta}(c) T, a_{1} \otimes a_{2}\right\rangle=\left\langle(T \circ \theta(c)) a_{1}, a_{2}\right\rangle=\left\langle\theta(c) a_{1}, T^{*} a_{2}\right\rangle .
$$

We may consider $A$ as a Banach right $A$-module with the product $b \cdot a:=\theta(a) b$. Since $\theta$ is non-degenerate, it follows that the linear span of the set $\{b \cdot a: a, b \in A\}$ is norm dense in $A$, and hence by Cohen's factorization theorem (Hewitt-Ross 25. Theorem 32.23]), for each $a \in A$, we may find $a^{\prime}, a^{\prime \prime} \in A$ such that $a=a^{\prime \prime} \cdot a^{\prime}=\theta\left(a^{\prime}\right) a^{\prime \prime}$. Therefore, writing $a_{1}=\theta\left(a_{1}^{\prime}\right) a_{1}^{\prime \prime}$, and continuing from (14), we have

$$
\begin{aligned}
\pi_{T, T_{*}}^{\theta}(c) & =\left\langle\theta(c)\left(\theta\left(a_{1}^{\prime}\right) a_{1}^{\prime \prime}\right), T^{*} a_{2}\right\rangle \\
& =\left\langle\theta\left(a_{1}^{\prime} c\right) a_{1}^{\prime \prime}, T^{*} a_{2}\right\rangle \\
& =\left\langle\theta\left(a_{1}^{\prime} c\right), a_{1}^{\prime \prime} \otimes T^{*} a_{2}\right\rangle \\
& =\left\langle c, \theta^{*}\left(a_{1}^{\prime \prime} \otimes T^{*} a_{2}\right) \cdot a_{1}^{\prime}\right\rangle .
\end{aligned}
$$

Hence $\pi_{T, T_{*}}^{\theta}=\theta^{*}\left(a_{1}^{\prime \prime} \otimes T^{*} a_{2}\right) \cdot a_{1}^{\prime} \in A^{*} \cdot A=L U C(A)$. 
In the general case that $\eta=\sum_{i=1}^{\infty} a_{i} \otimes b_{i} \in A \widehat{\otimes} A$, we let $\eta_{n}=\sum_{i=1}^{n} a_{i} \otimes b_{i}$, so that $\left\|\eta-\eta_{n}\right\| \rightarrow 0$ as $n \rightarrow \infty$. Then

$$
\begin{aligned}
\left\|\pi_{T, \eta}^{\theta}-\pi_{T, \eta_{n}}^{\theta}\right\| & =\sup _{\|c\| \leq 1}\left|\pi_{T, \eta}^{\theta}(c)-\pi_{T, \eta_{n}}^{\theta}(c)\right| \\
& =\sup _{\|c\| \leq 1}\left|\left\langle\pi^{\theta}(c) T, \eta-\eta_{n}\right\rangle\right| \\
& \leq\left\|\eta-\eta_{n}\right\| \sup _{\|c\| \leq 1}\|T \circ \theta(c)\| \\
& \leq\left\|\eta-\eta_{n}\right\|\|\theta\|\|T\| \rightarrow 0,
\end{aligned}
$$

as $n \rightarrow \infty$. Therefore $\pi_{T, \eta}^{\theta} \in L U C(A)$ for all $T \in \mathscr{L}\left(A, A^{*}\right), \eta \in A \widehat{\otimes} A$, completing the proof of subordination of $\pi^{\theta}$ to $L U C(A)$.

Let $\left(A^{\mathrm{op}}, *\right)$ be the opposite algebra to $A$ (that is, $a * b=b a$ ). Then by applying the above theorem to $A^{\mathrm{op}}$ and using the fact that $L U C\left(A^{\mathrm{op}}\right)=R U C(A)$, we obtain the following corollary.

Corollary 5.5. Let $A$ be a Banach algebra with a bounded left approximate identity. Let $\theta: A \longrightarrow \mathscr{L}(A)$ be a continuous, non-degenerate, representation. Then each of the two maps

(i) $\pi^{\theta}: A \longrightarrow \mathscr{L}\left(\mathscr{L}\left(A, A^{*}\right)\right), \quad \pi^{\theta}(a) T=T \circ \theta(a)$,

(ii) $\check{\pi}^{\theta}: A \longrightarrow \mathscr{L}\left(\mathscr{L}\left(A, A^{*}\right)\right), \quad \check{\pi}^{\theta}(a) T=\theta(a)^{*} \circ T$,

where $a \in A, T \in \mathscr{L}\left(A, A^{*}\right)$, is a continuous anti-representation of $A$ subordinate to $\operatorname{RUC}(A)$.

Next we prove an $L U C$-representation theorem for left uniformly continuous functionals. The theorem shows that all elements of $L U C(A)$ can be represented as the coordinate functions of each of the representations $\pi^{\theta}$ and $\check{\pi}^{\theta}$ in Theorem 5.4, for a suitable $\theta$.

Theorem 5.6. Let $A$ be a Banach algebra with a bounded right approximate identity, and let $\theta: A \longrightarrow \mathscr{L}(A)$ be the continuous anti-representation defined by $\theta(a)=$ $R_{a}$, with $R_{a}$ being the right multiplication operator by a. Then every $f \in L U C(A)=$ $A^{*} \cdot A$ is a coordinate function of each of the representations $\pi^{\theta}$ and $\check{\pi}^{\theta}$ of $A$ on $\mathscr{L}\left(A, A^{*}\right)$, defined by $\pi^{\theta}(a) T=T \circ \theta(a)$ and $\check{\pi}^{\theta}(a) T=\theta(a)^{*} \circ T$.

Proof. We give the proof for $\pi^{\theta}$; the case of $\check{\pi}^{\theta}$ is similar. Let $f=g \cdot a \in L U C(A)$, where $g \in A^{*}, a \in A$. Using Cohen's factorization theorem, let $a=a_{1} a_{2}$, with $a_{1}, a_{2} \in A$. We define $M_{g} \in \mathscr{L}\left(A, A^{*}\right)$ by $M_{g}(b)=b \cdot g(b \in A)$, and $T_{*} \in A \widehat{\otimes} A=$ $\mathscr{L}\left(A, A^{*}\right)_{*}$ by $T_{*}=a_{2} \otimes a_{1}$. We claim that $f=\pi_{M_{g}, T_{*}}^{\theta}$. In fact, for every $c \in A$,

$$
\begin{aligned}
\pi_{M_{g}, T_{*}}^{\theta}(c) & =\left\langle\pi^{\theta}(c) M_{g}, T_{*}\right\rangle=\left\langle M_{g} \circ \theta(c), a_{2} \otimes a_{1}\right\rangle=\left\langle M_{g}\left(a_{2} c\right), a_{1}\right\rangle \\
& =\left\langle\left(a_{2} c\right) \cdot g, a_{1}\right\rangle=\left\langle g,\left(a_{1} a_{2}\right) c\right\rangle=\langle g \cdot a, c\rangle=\langle f, c\rangle .
\end{aligned}
$$

By applying the above theorem to the opposite algebra $A^{\text {op }}$, we obtain the following analogous result for $R U C(A)$. 
Corollary 5.7. Let $A$ be a Banach algebra with a bounded left approximate identity, and let $\theta: A \longrightarrow \mathscr{L}(A)$ be the continuous representation defined by $\theta(a)=L_{a}$, with $L_{a}$ being the left multiplication operator by a. Then every $f \in R U C(A)=A \cdot A^{*}$ is a coordinate function of each of the continuous anti-representations $\pi^{\theta}$ and $\check{\pi}^{\theta}$ of $A$ on $\mathscr{L}\left(A, A^{*}\right)$, defined by $\pi^{\theta}(a) T=T \circ \theta(a)$ and $\check{\pi}^{\theta}(a) T=\theta(a)^{*} \circ T$.

\section{ACKNowledgements}

We would like to thank the referee for the useful comments and suggestions and for bringing the references 22,29] to our attention.

Large parts of the paper were written during the visits of the first author to Windsor, Carleton and Waterloo Universities in 2011 and 2013. He would like to thank Mehdi Monfared, Matthias Neufang and Nico Spronk for their hospitality, as well as all the folks at each department of mathematics. His visits were partially supported by NSERC grants of the hosts. This support is gratefully acknowledged.

\section{REFERENCES}

[1] Richard Arens, The adjoint of a bilinear operation, Proc. Amer. Math. Soc. 2 (1951), 839-848. MR0045941 (13,659f)

[2] Richard Arens, Operations induced in function classes, Monatsh. Math. 55 (1951), 1-19. MR0044109 (13,372b)

[3] Bernard Beauzamy, Espaces d'interpolation réels: topologie et géométrie (French), Lecture Notes in Mathematics, vol. 666, Springer, Berlin, 1978. MR513228 (80k:46080)

[4] F. F. Bonsall and J. Duncan, Dual representations of Banach algebras, Acta Math. 117 (1967), 79-102. MR0205094 (34 \#4929)

[5] F. F. Bonsall and J. Duncan, Dually irreducible representations of Banach algebras, Quart. J. Math. Oxford Ser. (2) 19 (1968), 97-111. MR0232209 (38 \#535)

[6] Frank F. Bonsall and John Duncan, Complete normed algebras, Springer-Verlag, New YorkHeidelberg, 1973. Ergebnisse der Mathematik und ihrer Grenzgebiete, Band 80. MR0423029 (54 \#11013)

[7] Nicolas Bourbaki, Integration. I. Chapters 1-6, Elements of Mathematics (Berlin), SpringerVerlag, Berlin, 2004. Translated from the 1959, 1965 and 1967 French originals by Sterling K. Berberian. MR2018901 (2004i:28001)

[8] Paul Civin and Bertram Yood, The second conjugate space of a Banach algebra as an algebra, Pacific J. Math. 11 (1961), 847-870. MR0143056 (26 \#622)

[9] H. G. Dales, Banach algebras and automatic continuity, London Mathematical Society Monographs. New Series, vol. 24, The Clarendon Press, Oxford University Press, New York, 2000. Oxford Science Publications. MR 1816726 (2002e:46001)

[10] H. G. Dales and A. T.-M. Lau, The second duals of Beurling algebras, Mem. Amer. Math. Soc. 177 (2005), no. 836, vi+191, DOI 10.1090/memo/0836. MR2155972 (2006k:43002)

[11] W. J. Davis, T. Figiel, W. B. Johnson, and A. Pełczyński, Factoring weakly compact operators, J. Functional Analysis 17 (1974), 311-327. MR0355536 (50 \#8010)

[12] Matthew Daws, Dual Banach algebras: representations and injectivity, Studia Math. 178 (2007), no. 3, 231-275, DOI 10.4064/sm178-3-3. MR2289356 (2008f:46062)

[13] Antoine Derighetti, Mahmoud Filali, and Mehdi Sangani Monfared, On the ideal structure of some Banach algebras related to convolution operators on $L^{p}(G)$, J. Funct. Anal. 215 (2004), no. 2, 341-365, DOI 10.1016/j.jfa.2004.02.011. MR2151297(2008b:43014)

[14] Jacques Dixmier, Les $C^{*}$-algèbres et leurs représentations (French), Les Grands Classiques Gauthier-Villars. [Gauthier-Villars Great Classics], Éditions Jacques Gabay, Paris, 1996. Reprint of the second (1969) edition. MR.1452364 (98a:46066) 
[15] J. Duncan and A. Ülger, Almost periodic functionals on Banach algebras, Rocky Mountain J. Math. 22 (1992), no. 3, 837-848, DOI 10.1216/rmjm/1181072699. MR/1183691 (93h:46057)

[16] Nelson Dunford and Jacob T. Schwartz, Linear operators. Part I: General theory; With the assistance of William G. Bade and Robert G. Bartle; Reprint of the 1958 original; A WileyInterscience Publication, Wiley Classics Library, John Wiley \& Sons, Inc., New York, 1988. MR.1009162 (90g:47001a)

[17] J. M. G. Fell, The dual spaces of Banach algebras, Trans. Amer. Math. Soc. 114 (1965), 227-250. MR0172131 (30 \#2357)

[18] M. Filali and M. Sangani Monfared, Finite-dimensional left ideals in the duals of introverted spaces, Proc. Amer. Math. Soc. 139 (2011), no. 10, 3645-3656, DOI 10.1090/S0002-99392011-10784-6. MR2813394 (2012e:46096)

[19] Gerald B. Folland, A course in abstract harmonic analysis, Studies in Advanced Mathematics, CRC Press, Boca Raton, FL, 1995. MR1397028 (98c:43001)

[20] J. Galindo, On group and semigroup compactification of topological groups, preprint.

[21] F. Ghahramani, Isometric representation of $M(G)$ on $B(H)$, Glasgow Math. J. 23 (1982), no. 2, 119-122, DOI 10.1017/S0017089500004882. MR663137 (83m:43002)

[22] Mahya Ghandehari, Amenability properties of Rajchman algebras, Indiana Univ. Math. J. 61 (2012), no. 3, 1369-1392, DOI 10.1512/iumj.2012.61.4622. MR.3071703

[23] E. E. Granirer, On some spaces of linear functionals on the algebras $A_{p}(G)$ for locally compact groups, Colloq. Math. 52 (1987), no. 1, 119-132. MR891504 (88k:43006)

[24] Edwin Hewitt and Kenneth A. Ross, Abstract harmonic analysis. Vol. I, 2nd ed., Grundlehren der Mathematischen Wissenschaften [Fundamental Principles of Mathematical Sciences], vol. 115, Springer-Verlag, Berlin-New York, 1979. Structure of topological groups, integration theory, group representations. MR551496 (81k:43001)

[25] Edwin Hewitt and Kenneth A. Ross, Abstract harmonic analysis. Vol. II: Structure and analysis for compact groups. Analysis on locally compact Abelian groups, Die Grundlehren der mathematischen Wissenschaften, Band 152, Springer-Verlag, New York-Berlin, 1970. MR.0262773 (41 \#7378)

[26] Sten Kaijser, On Banach modules. I, Math. Proc. Cambridge Philos. Soc. 90 (1981), no. 3, 423-444, DOI 10.1017/S0305004100058904. MR628827 (83f:46057)

[27] Anthony To Ming Lau, Uniformly continuous functionals on the Fourier algebra of any locally compact group, Trans. Amer. Math. Soc. 251 (1979), 39-59, DOI 10.2307/1998682. MR.531968(80m:43009)

[28] Anthony To Ming Lau, Uniformly continuous functionals on Banach algebras, Colloq. Math. 51 (1987), 195-205. MR891287 (88f:43006)

[29] Anthony To-Ming Lau and J. Ludwig, Fourier-Stieltjes algebra of a topological group, Adv. Math. 229 (2012), no. 3, 2000-2023, DOI 10.1016/j.aim.2011.12.022. MR2871165 (2012m:43004)

[30] Michael Megrelishvili, Fragmentability and representations of flows, Proceedings of the 17th Summer Conference on Topology and its Applications, Topology Proc. 27 (2003), no. 2, 497-544. MR2077804 (2005h:37042)

[31] Theodore W. Palmer, Banach algebras and the general theory of ${ }^{*}$-algebras. Vol. I, Encyclopedia of Mathematics and its Applications, vol. 49, Cambridge University Press, Cambridge, 1994. Algebras and Banach algebras. MR1270014 (95c:46002)

[32] Volker Runde, Amenability for dual Banach algebras, Studia Math. 148 (2001), no. 1, 47-66, DOI 10.4064/sm148-1-5. MR1881439(2002m:46078)

[33] Volker Runde, Dual Banach algebras: Connes-amenability, normal, virtual diagonals, and injectivity of the predual bimodule, Math. Scand. 95 (2004), no. 1, 124-144. MR2091485 (2006a:46059)

[34] Roger R. Smith and Nico Spronk, Representations of group algebras in spaces of completely bounded maps, Indiana Univ. Math. J. 54 (2005), no. 3, 873-896, DOI 10.1512/iumj.2005.54.2551. MR2151237(2006c:46047)

[35] Erling Størmer, Regular abelian Banach algebras of linear maps of operator algebras, J. Funct. Anal. 37 (1980), no. 3, 331-373, DOI 10.1016/0022-1236(80)90048-8. MR.581427(81k:46057)

[36] M. Takesaki, Theory of operator algebras. I: Operator Algebras and Non-commutative Geometry, 5, Encyclopaedia of Mathematical Sciences, vol. 124, Springer-Verlag, Berlin, 2002. Reprint of the first (1979) edition. MR,1873025 (2002m:46083) 
[37] N. J. Young, The irregularity of multiplication in group algebras, Quart J. Math. Oxford Ser. (2) 24 (1973), 59-62. MR0320756 (47 \#9290)

[38] N. J. Young, Periodicity of functionals and representations of normed algebras on reflexive spaces, Proc. Edinburgh Math. Soc. (2) 20 (1976/77), no. 2, 99-120. MR0435849(55 \#8800)

Department of Mathematical Sciences, University of Oulu, Oulu 90014, Finland

E-mail address: mahmoud.filali@oulu.fi

School of Mathematics and Statistics, Carleton University, Ottawa, Ontario K1S 5B6, Canada - And - Université Lille 1, U.F.R. de Mathématiques, Laboratoire Paul Painlevé, 59655 Villeneuve D'AscQ, France

E-mail address: mneufang@math.carleton.ca

Department of Mathematics and Statistics, University of Windsor, Windsor, Ontario N9B 3P4, CANADA

E-mail address: monfared@uwindsor.ca 